\title{
Uncharakteristisches Fieber (UF), afebrile Allgemeinreaktion (AFAR), Luftwegekatarrhe, Tonsillitis
}

1.1 Prozessgerechte Klassifizierung - 31

1.2 Uncharakteristisches Fieber (UF) - 32

1.2.1 Fachsprache, Berufsjargon und Laienausdrücke -32

1.2.2 Erregernachweis - 34

1.2.3 Verläufe -35

1.2.4 Bettlägerigkeit und Hausbesuch -35

1.2.5 Höhe der Körpertemperatur - 36

1.2.6 Subjektives und objektives Befinden -36

1.2.7 Wochenlange Temperaturerhöhungen -37

1.2.8 Nicht mehr uncharakteristisch -37

1.2.9 Qualitätskontrolle am Beispiel des Fieber-Programms - 37

1.2.10 Management - 39

1.3 Afebrile Allgemeinreaktion (AFAR) -40

1.4 Afebriler Husten - 40

1.4.1 Intuitive primäre Diagnostik -41

1.4.2 Verschlimmerung -41

1.4.3 Symptomgruppe »Bronchitis« -41

1.4.4 Keuchhusten -42

1.5 Halsschmerzen - 42

1.5.1 Tonsillitis -42

1.5.2 Infektiöse Mononukleose -43

1.6 Heiserkeit -44

1.7 Schnupfen -44

1.7.1 Inanspruchnahme des Arztes - 45

1.7.2 Verlauf -45

1.8 Kombinierte Luftwegekatarrhe $\quad-45$

1.9 Kruppbilder -45

1.10 Fieberkrampf -46

Thematik des Fachgesprächs $\quad-47$ 
In Kap. 1 werden jene Fälle behandelt, die diagnostisch und therapeutisch eng zusammengehören und sich durch ihre überragende Häufigkeit auszeichnen. Dazu gehören:

- uncharakteristisches Fieber (UF),

- gleichartige, jedoch afebrile Verläufe (afebrile Allgemeinreaktion/AFAR),

- Angina tonsillaris (die häufig genug das Symptom einer viralen Allgemeinerkrankung darstellt), sowie

- verschiedene fieberfreie Katarrhe der Luftwege (•Tabelle 1.1).

\subsection{Prozessgerechte Klassifizierung}

Der Allgemeinarzt sollte sich bei jeder Konsultation (sowohl bei verschiedenen Fällen als auch im Verlauf desselben Falles) am Beratungsende darüber im Klaren sein, welcher Art das gewonnene BE ist:

- Symptom (A),

- Symptomgruppe (B),

- Bild einer Krankheit (C),

- exakte Diagnose (D).

Ein Blick in die zweidimensionale Systematik (• Tabelle A2, S. 18 zeigt, dass für die BEs des Fensters 1 eine exak- te Diagnose (D) nur in einem einzigen von 1.168 Fällen (o,09\%) möglich war. Die Klassifizierung von Symptomgruppen (B) überwiegt eindeutig.

Die Zuordnung der einzelnen Beratungsergebnisse zu A bis D ist nicht nur unter fällestatistischen Gesichtspunkten zu betrachten, sondern hat auch für die Denk- und Vorgehensweise des Allgemeinarztes wesentliche Konsequenzen:

(? Erkrankungen, die nach A, B oder C klassifiziert wurden, müssen abwartend offen geführt werden, d. h. der Arzt muss bereit sein, das BE zu revidieren, sobald besondere Symptome dazutreten, oder wenn der Verlauf der Erkrankung in eine ungewöhnliche Richtung weist.

Wurde durch den Arzt jedoch eine exakte Diagnose gestellt (D), dann brauchen primär keine weiteren diagnostischen Überlegungen mehr vorgenommen zu werden. In diesen Fällen (z. B. Angina tonsillaris mit Erregernachweis) ist nach den Vorschriften der spezialistischen Lehre zu behandeln.

Eine »dia" -"gnosis" im Sinne eines »Durchschauens bis auf den Grund « ist für nahezu alle BEs des Fensters 1 im allgemeinmedizinisch-wissenschaftlichen Sinn unmöglich. Hier kann der Arzt in der Praxis der Lehre nicht

Tabelle 1.1. Häufigkeit (Rang) der Beratungsergebnisse »uncharakteristisches Fieber (UF), afebrile Allgemeinreaktion (AFAR), Luftwegekatarrhe, Tonsillitis« in allgemeinmedizinischen Praxen in Österreich und in der Schweiz

\begin{tabular}{|c|c|c|c|}
\hline \multirow[t]{2}{*}{ Beratungsergebnisse } & \multicolumn{2}{|l|}{$\begin{array}{l}\text { Österreich } \\
\text { Braun }\end{array}$} & \multirow{2}{*}{$\begin{array}{l}\begin{array}{l}\text { Schweiz } \\
\text { Landolt-Theus }\end{array} \\
\text { 1983-1988 }\end{array}$} \\
\hline & 1954-1959 & 1977-1980 & \\
\hline UF & 1 & 1 & 2 \\
\hline AFAR & 24 & 10 & 3 \\
\hline Angina tonsillaris & 9 & 33 & 14 \\
\hline Husten, afebril & 3 & 6 & 20 \\
\hline Rhinitis, afebril (Schnupfen) & 47 & 35 & 21 \\
\hline Halsschmerzen, afebril & 78 & 53 & 39 \\
\hline Luftwegekatarrhe, afebrile (obere und/oder untere Luftwege) & 29 & 17 & 42 \\
\hline Pharyngitis, afebril & 20 & 23 & 61 \\
\hline Zustand nach UF & 23 & 92 & 85 \\
\hline Laryngitis, afebril (Heiserkeit) & 85 & 68 & 152 \\
\hline Bronchitis, afebril & 56 & 141 & 161 \\
\hline Temperaturerhöhung wochenlang & 290 & 286 & 239 \\
\hline Fieber mit auffälligen Myalgien & - & $336^{\mathrm{a}}$ & $412^{\mathrm{a}}$ \\
\hline Impffieber & 235 & $347^{\mathrm{a}}$ & $430^{\mathrm{a}}$ \\
\hline
\end{tabular}

${ }^{a}$ Nicht regelmäßig häufig 
folgen, auch wenn er sich aufgrund der bisherigen Universitätsausbildung verpflichtet fühlt, seine Fälle als erkannte Krankheiten auszuweisen.

\subsection{Uncharakteristisches Fieber (UF)}

In der modernen Allgemeinmedizin ist ein Bedarf an wissenschaftlich brauchbaren Begriffen unübersehbar. So hat sich im Falle der sog. Erkältungskrankheiten die Suche nach passenden diagnostischen Begriffen aufgedrängt. Braun hat daher aus der Praxisforschung heraus u. a. 2 neue Begriffe in die allgemeinmedizinische Fachsprache eingeführt:

- uncharakteristisches Fieber (UF) und

- afebrile Allgemeinreaktion (AFAR).

Diese beiden Begriffe werden noch nicht allgemein gebraucht, obwohl sie wissenschaftlich fundiert sind.

Es handelt sich bei diesen BEs nicht um Diagnosen (D), sondern um die Klassifizierung eines Symptoms (A) oder um die Klassifizierung einer Symptomgruppe (B).

Diese Begriffe drücken aus, dass keine herkömmliche "Diagnose« gemeint ist. Uncharakteristisches Fieber und afebrile Allgemeinreaktion gehen von Symptomen aus, in die nichts Spekulatives hineininterpretiert ist. Die Bezeichnungen UF und AFAR sind den Situationen in der Allgemeinpraxis angepasst, d.h. sie spiegeln die in der Praxis herrschende diagnostische Lage wider. Dadurch wurde eine zwanglose, wissenschaftlich vertretbare Benennung solcher Fälle möglich.

Uncharakteristisches Fieber und afebrile Allgemeinreaktion nehmen in verschiedenen Untersuchungen aus allgemeinmedizinischen Praxen, die über viele Jahre hinweg zu unterschiedlichen Zeitpunkten durchgeführt wur- den, in überraschender Auffälligkeit die weitaus vordersten Ränge in der Häufigkeit ein (• Tabelle A1 und 1.2). In - Tabelle 1.2 ist wiederum die Verteilung auf die einzelnen Altersgruppen bemerkenswert.

\subsubsection{Fachsprache, Berufsjargon und Laienausdrücke}

In der allgemeinmedizinischen Fachsprache (Kasugraphie) ist UF wie folgt definiert:

- Fieber mit Allgemeinsymptomen, ggf. auch mit lokalen Symptomen.

Zu den Allgemein- und Lokalsymptomen gehören obligat Fieber, fakultativ Mattigkeit, Müdigkeit, Appetitlosigkeit, Schlafstörungen, Frösteln, Schweißausbrüche, uncharakteristischer Ausschlag. Ferner: z. B. Schnupfen, Niesen, Halsschmerzen, Husten, Kopfschmerzen, Gliederschmerzen, Erbrechen, Durchfall, Pollakisurie.

Die angeführten Beschwerden und Erscheinungen treten in verschiedenster Kombination auf; hierbei schwankt deren Zahl außerordentlich. Sie sind meist nur leicht ausgeprägt und flüchtig und dauern in der Regel einige Tage, in Einzelfällen bis zu 2 Wochen.

R. Klein hat in seiner Allgemeinpraxis während der Wintermonate 2000/2001 und 2001/2002 121 bzw. 62 Patienten mithilfe der $\triangleright$ Checkliste Nr. 1 ( $\triangleright$ Übersicht 1.1) programmiert untersucht. Erwartungsgemäß wurden von seinen Patienten mit uncharakteristischem Fieber Allgemeinsymptome, wie Frösteln, Schwitzen, Mattigkeit, Appetitlosigkeit, Bettlägerigkeit und Schlafstörungen, bevorzugt geklagt (• Abb. 1.1). Daneben wurde häufig von Husten mit und ohne Auswurf, Schnupfen sowie Muskel-, Gelenk- und Halsschmerzen berichtet.

- Tabelle 1.2. Häufigkeit (Rang) der Beratungsergebnisse »uncharakteristisches Fieber« (UF) und afebrile Allgemeinreaktion« (AFAR) in allgemeinmedizinischen Praxen in Österreich der Jahre 1977-1980, aufgeschlüsselt nach Geschlecht und Altersgruppe, im Vergleich zum Vierjahresmaterial der Jahre 1955-1959

\begin{tabular}{|c|c|c|c|c|c|c|c|c|c|c|c|}
\hline \multicolumn{2}{|c|}{ 1977-1980 } & \multicolumn{2}{|c|}{ 1954-1959 } & \multirow{3}{*}{$\begin{array}{l}\text { Beratungs- } \\
\text { ergebnis }\end{array}$} & \multicolumn{7}{|c|}{$1977-1980$} \\
\hline \multirow[t]{2}{*}{ Rang } & \multirow[t]{2}{*}{$\%$ o] } & \multirow[t]{2}{*}{ Rang } & \multirow[t]{2}{*}{ [\%o] } & & \multirow{2}{*}{$\begin{array}{l}\text { Gesamt } \\
\text { (n) }\end{array}$} & \multirow[t]{2}{*}{ Männlich } & \multirow[t]{2}{*}{ Weiblich } & \multicolumn{4}{|c|}{ Altersgruppen [Jahre] } \\
\hline & & & & & & & & $0-14$ & $15-44$ & $45-64$ & $\geq 65$ \\
\hline 1 & 52,7 & 1 & 80,4 & UF & 419 & 242 & 177 & 203 & 134 & 55 & 27 \\
\hline 10 & 20,5 & 24 & 8,1 & AFAR & 163 & 62 & 101 & 16 & 75 & 54 & 18 \\
\hline
\end{tabular}




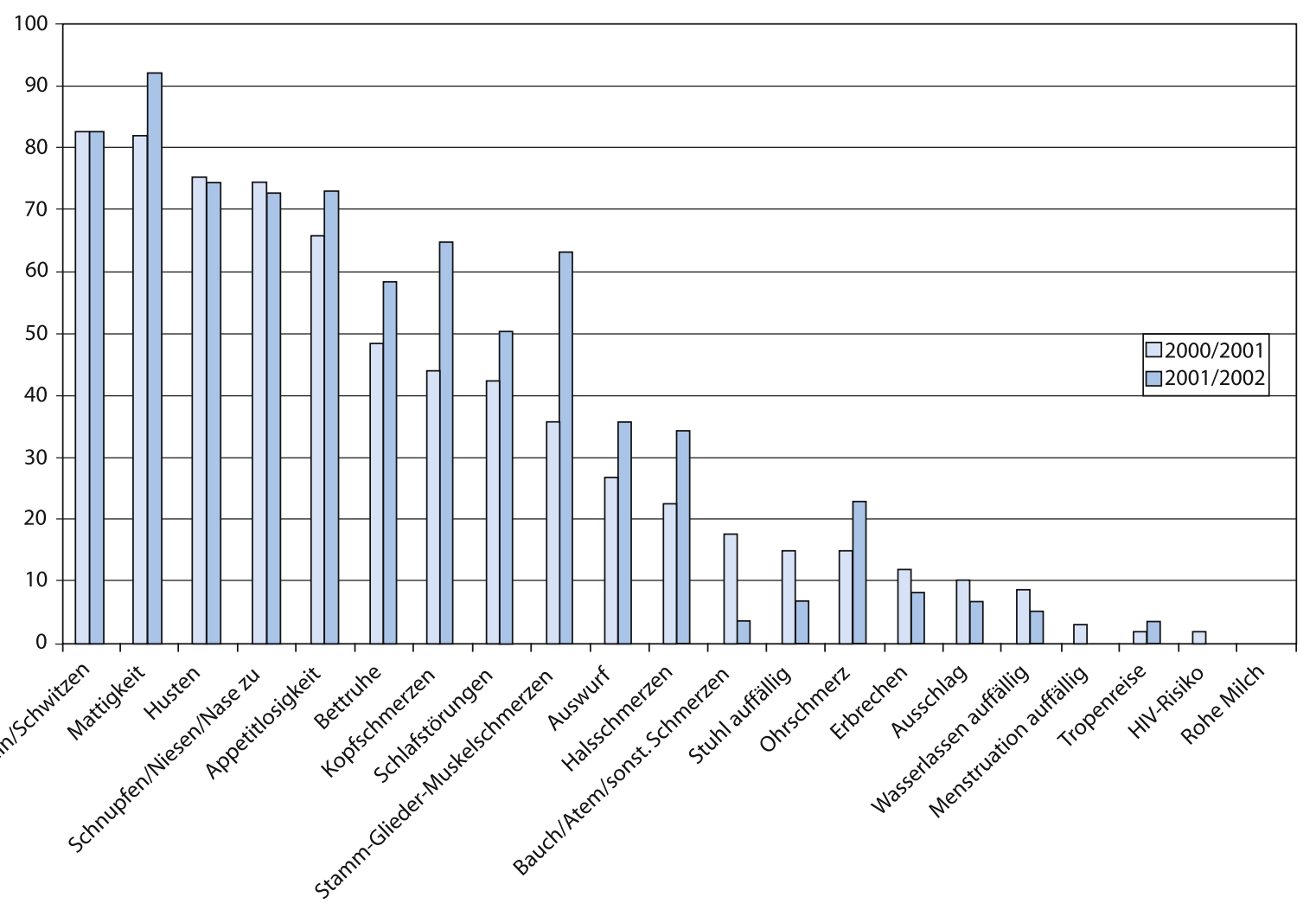

- Abb. 1.1. Häufigkeitsverteilung der Patientenangaben bei uncharakteristischem Fieber (Klein 2003)

Bei den bei der körperlichen Untersuchung erhobenen Befunden standen in beiden Beobachtungszeiträumen Symptome im Kopfbereich weit im Vordergrund (• Abb. 1.2). Pathologien im Mund- und Rachenbereich, Schwellung im Halsbereich, Symptome, die auf Affektionen der Nebenhöhlen hindeuten, sowie katharralische Sekretion machten den Löwenanteil der erhobenen Befunde aus. In der Saison 2000/2001 waren pathologische Lungenauskultationsbefunde besonders häufig (Klein 2003).

Im ärztlichen Berufsjargon und in der Laienwelt existieren zahlreiche Ausdrücke, die von Land zu Land in unterschiedlicher, individueller Bedeutung als sog. Diagnosen verwendet werden, z. B. grippaler Infekt, Grippe, Virusinfekt (Bauch-, Kopf-)Grippe, Influenza, (fieberhafte) Bronchitis, Fieberzustand, Verkühlung, Erkältung.

Diese Begriffe sind z. T. Ausdruck eines Kausalitätsbedürfnisses des Patienten (»Ich bin gestern nass geworden...«), z. T. aber auch von Ärzten.

Der Erkältungsbegriff hat in der praktisch angewandten Medizin v. a. die Bedeutung einer dem Patienten plau- sibel erscheinenden Ausrede bei vielen ursächlich unklaren Krankheitsfällen.

Bei den Ärzten können sozialversicherungsrechtliche Erfordernisse, aber auch ärztlicher Ehrgeiz die Ursache dafür sein, "Diagnosen « auszuweisen, oder einfach die unausgesprochene Angst, vor dem Kollegen ohne »Diagnose» dazustehen.

Letztlich bedient sich der Allgemeinarzt dieser Jargonsprache (z. B. grippaler Infekt, akute respiratorische Erkrankung, ARI oder akut auftretende Infektionskrankheit der Atemwege, AIA), weil er während seiner Ausbildung nicht mit praxisgerechten Begriffen im Hinblick auf eine spätere Tätigkeit als Allgemeinarzt ausgestattet worden ist. Unabhängig von der Diskussion um eine korrekte fachsprachliche Bezeichnung wird es jedoch stets die Herausforderung des qualifiziert arbeitenden Allgemeinarztes bleiben, durch gezielte Befragung, programmierte Diagnostik und körperliche Untersuchung in der Regel eine schwere, bedrohliche Krankheit zu bedenken und selten einmal durch weitere diagnostische Verfahren 


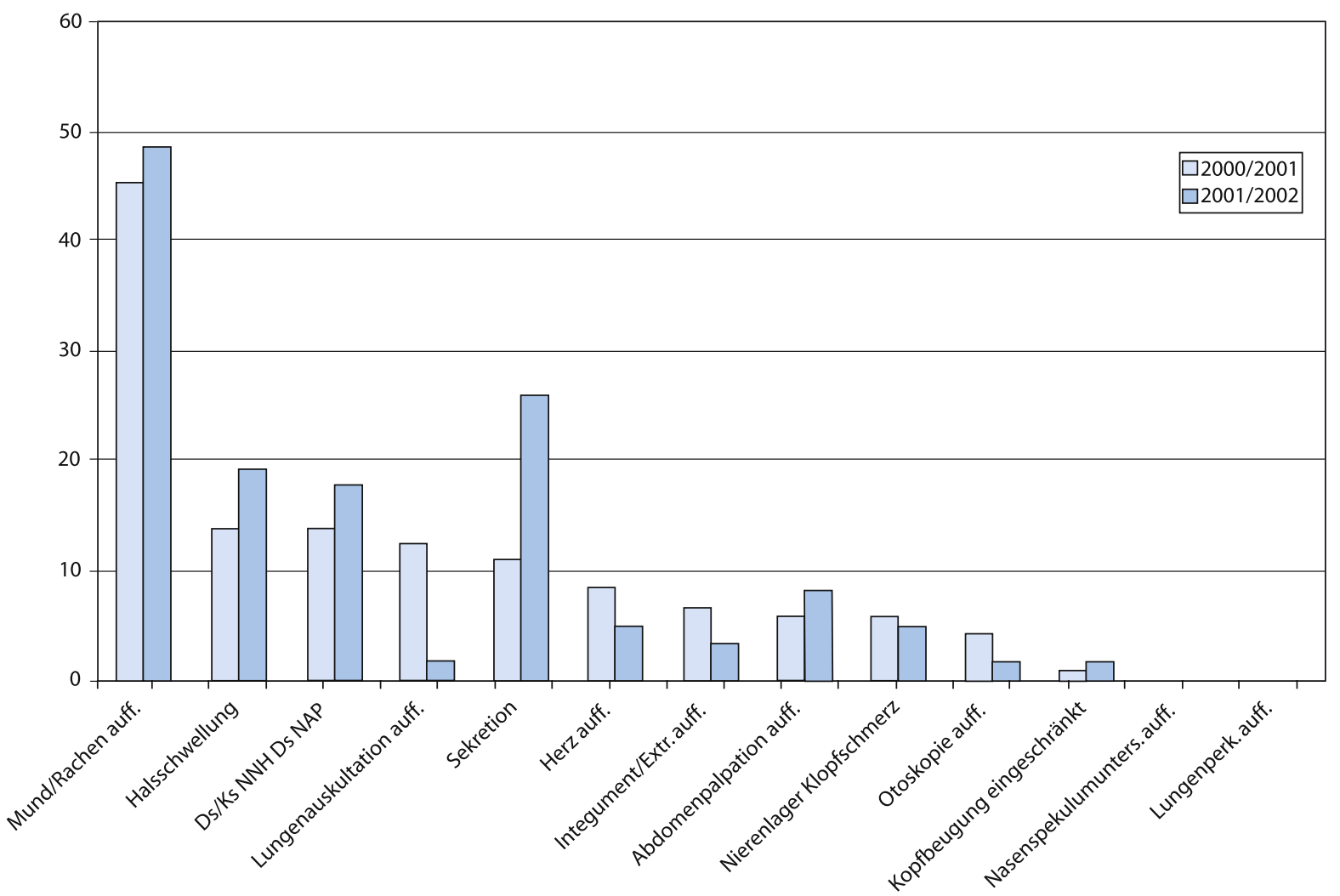

- Abb. 1.2. Häufigkeitsverteilung der objektiven Befunde bei uncharakteristischem Fieber (Klein 2003)

mögliche abwendbar gefährlichen Verläufe (Otitis media, Sinusitis, Pneumonie, Phlegmone/Abszedierung, Meningitis) auszuschließen.

\subsubsection{Erregernachweis}

Von sämtlichen fieberhaften Erkrankungen ist das UF mit schätzungsweise $50-60 \%$ das häufigste Beratungsergebnis in der Allgemeinpraxis. Den hohen Rang bestätigen die Fällestatistiken von Braun (1961), Göpel (1975), Prosénc (1967), Landolt-Theus (1992) sowie Danninger (1997).

Untersuchungen haben immer wieder ergeben, dass in mindestens der Hälfte der unspezifischen Fieberfälle der direkte und der indirekte Erregernachweis versagen. Der Erregernachweis ist wegen der enormen Häufigkeit der Fälle ohnedies nicht finanzierbar und wegen der sehr guten Prognostik des Durchschnittsfalls auch nicht nötig.
Er muss allerdings dann verlangt werden, wenn die Begriffe Grippe, Influenza o. Ä. korrekt verwendet werden. Einzelne Pandemien (Grippepandemien: 1918/19 "Spanische Grippe«, 1957 »Asiatische Grippe«, 1968/69 "Hongkong-Grippe«) wurden virologisch bestimmten Influenzavirustypen zugeordnet.

Die Zahl der Erreger, die für eine Infektion des Respirationstrakts in Frage kommen, ist so groß und das klinische Erscheinungsbild der durch die verschiedenen Erreger ausgelösten Erkrankungen so wenig charakteristisch, dass sich eine exakte Diagnose (D) nur aufgrund virologischer und serologischer Untersuchungen stellen lässt.

Dies gilt auch für das 2003 entdeckte Coronavirus als Erreger des schweren akuten respiratorischen Syndroms (SARS).

Im Übrigen können beim UF auch nach intensiver und extensiver Testung nur in weniger als der Hälfte der Fälle pathogene Organismen (Viren oder Bakterien) isoliert werden. 
- Tabelle 1.3. Symptome und deren Ausprägung im Hinblick auf die Zuordnung »eher Influenza« bzw. »eher Erkältung«

\begin{tabular}{l|l|l|}
\hline Symptome & Eher Influenza & Eher Erkältung \\
\hline Schnupfen & + & ++ \\
Kopfschmerzen & +++ & +++ \\
Muskel-/Gliederschmerzen & +++ & + \\
Halsschmerzen & + & + \\
Abgeschlagenheit/Unwohlsein & +++ & + \\
Husten & +++ & ++ \\
Frösteln & +++ & ++ \\
Fieber & +++ & ++ \\
& & -
\end{tabular}

Das Vorhandensein bestimmter Symptome (z. B. Fieber und Husten) bzw. deren Ausprägung kann in einzelnen Fällen einen Hinweis darauf geben, ob es der Hausarzt mit einem Patienten zu tun hat, der eine Influenza hat, oder ob eher eine Erkältung vorliegt (• Tabelle 1.3).

Epidemien von UF sind nicht an eine bestimmte Jahreszeit gebunden.

Die Morbidität (definiert als die Häufigkeit des Auftretens einer bestimmten Krankheit, bezogen auf die Gesamtpopulation) ist bei Kindern und Jugendlichen während Influenzaepidemien immer höher als bei Erwachsenen, da dieser Altersgruppe die Basisimmunität fehlt.

Die Letalität (definiert als der Anteil der Erkrankten, die sterben) ist jedoch bei älteren Personen relativ hoch.

\subsubsection{Verläufe}

Der Arzt kann zum Zeitpunkt der Untersuchung oft nicht abschätzen, ob sich der Patient am Beginn, am Höhepunkt oder im Stadium der bereits abklingenden Erkrankung befindet.

Das UF kann verschiedene Verläufe nehmen:

- Die Symptome verschwinden innerhalb von Stunden und Tagen.

- Neue uncharakteristische Symptome kommen dazu.

- Charakteristische Symptome (z. B.ein pneumonischer Auskultationsbefund, eine »eitrige« Tonsillitis) werden fassbar.

- Ein AGV entwickelt sich innerhalb von wenigen Stunden (z. B. Appendicitis acuta, Meningitis acuta, Sepsis, Pneumonie).
(D) Fallstricke bei fieberhaften Erkrankungen stellen z. B. atypische Appendizitiden oder Tropenkrankheiten (z. B. Malaria) dar.

\subsubsection{Bettlägerigkeit und Hausbesuch}

Der einzelne Patient oder seine Angehörigen reagieren auf das plötzliche Auftreten von Fieber (als einziges Symptom) durchaus unterschiedlich:

So wird z. B. ein im Erwerbsleben stehender Erwachsener $\mathrm{zu}$ einem üblichen "Grippemittel " greifen oder sich auf andere bereits erprobte Hausmittel verlassen. Die junge Mutter, deren erstes Kind fiebert, wird dagegen rasch den Arzt konsultieren. Hier kann (nach erfolgter Untersuchung) manchmal der Hinweis beruhigend wirken, dass 10-12 Fieberattacken im Jahr bis zum 10. Lebensjahr durchaus noch "normal« sein können.

Wenn sich der Berufstätige entschließt, den Arzt aufzusuchen, geht es ihm auch um die Verordnung von Arbeitsruhe, während die besorgte Mutter "Schlimmes" ausgeschlossen wissen möchte. Andererseits sind Mütter mit mehreren Kindern aufgrund ihrer Erfahrung durchaus bereit, zunächst ohne Arztkontakt auszukommen und sich auf bewährte Methoden (z. B. Wickel, Fieberzäpfchen) zu verlassen; dies besonders dann, wenn weitere Fieberfälle in der Umgebung bekannt sind.

In unserem Sozialversicherungssystem ruft man den Arzt zu Fiebernden überwiegend ins Haus. Dabei handelt es sich meist um Kinder vom 1. Lebensjahr bis zum Schulalter sowie um 20- bis 30-Jährige (•Tabelle 1.4).

Manche Patienten suchen aber auch die Sprechstunde auf oder lassen sich in die Praxis bringen, wenn es der Arzt 
Tabelle 1.4. Alters- und Geschlechtsverteilung von 745 wegen fieberhafter Zustandsbilder durch den dringlichen Hausbesuchsdienst (DHD) versorgten Patienten. (Handschak 1986)

\begin{tabular}{|c|c|c|c|c|}
\hline Altersgruppe & $\begin{array}{l}\mathrm{n} \\
\text { Männlich }\end{array}$ & $\begin{array}{l}n \\
\text { Weiblich }\end{array}$ & $\begin{array}{l}\mathrm{n} \\
\text { Gesamt }\end{array}$ & [\%] \\
\hline$\leq 3$ Monate & 2 & 2 & 4 & 0,5 \\
\hline 3 Monate-1 Jahr & 35 & 31 & 66 & 8,9 \\
\hline 1-3 Jahre & 76 & 56 & 132 & 17,7 \\
\hline 5-7 Jahre & 60 & 74 & 134 & 18,0 \\
\hline 7-14 Jahre & 31 & 26 & 57 & 7,7 \\
\hline 14-20 Jahre & 33 & 28 & 61 & 8,2 \\
\hline 20-30 Jahre & 39 & 50 & 89 & 11,9 \\
\hline 30-40 Jahre & 24 & 22 & 46 & 6,2 \\
\hline 40-50 Jahre & 20 & 14 & 34 & 4,6 \\
\hline 50-65 Jahre & 26 & 36 & 62 & 8,3 \\
\hline 65-80 Jahre & 25 & 22 & 47 & 6,3 \\
\hline$\geq 80$ Jahre & 7 & 6 & 13 & 1,7 \\
\hline Gesamt & 378 & 367 & 745 & 100,0 \\
\hline
\end{tabular}

vorschlägt. Auch bei Säuglingen und Kleinkindern mit hohem Fieber ist es durchaus zumutbar, diese gut geschützt zum Arzt fahren zu lassen.

\subsubsection{Höhe der Körpertemperatur}

Die Körpertemperatur kann an verschiedenen Stellen gemessen werden: rektal (am genauesten), vaginal, sublingual, axillär (ungenau) oder im Gehörgang.

Die rektal (oder vaginal) gemessene Temperatur (Goldstandard) liegt etwa $0,5^{\circ} \mathrm{C}$ höher als die axilläre Temperatur; sublingual werden um $0,2-0,3^{\circ} \mathrm{C}$ geringere Werte als rektal gemessen. Der übliche Temperaturbereich beim Gesunden bewegt sich zwischen $36^{\circ} \mathrm{C}$ und $37,5^{\circ} \mathrm{C}$ (rektal), Werte über $37,5^{\circ} \mathrm{C}$ rektal werden allgemein als erhöhte Temperatur bezeichnet.

Die per Infrarotmethode gemessene Ohrtemperatur steht zwar im Zusammenhang mit Temperaturen, die an anderen Körperstellen gemessen werden, ist aber nicht direkt mit diesen vergleichbar. Der Normalwert liegt mit dieser Methode für alle Altersstufen zwischen $35,8^{\circ} \mathrm{C}$ und $38^{\circ} \mathrm{C}$.

$\mathrm{Zu}$ beachten ist die dem Organismus angeborene Tagesperiodik der Körpertemperatur; die morgendliche Temperatur liegt $0,5-1^{\circ} \mathrm{C}$ unter der abendlichen. Eine andere zyklisch wiederkehrende Körpertemperaturände- rung tritt im Laufe des Menstruationszyklus der Frau auf (• Abb. 9.13). Darüber hinaus gibt es individuell deutliche Unterschiede (Streubereich um $1^{\circ} \mathrm{C}$ ).

Die normale Körpertemperatur hängt auch vom Alter ab. Die Normaltemperatur liegt bei Babys und Kleinkindern am höchsten. Mit ungefähr 11 Jahren beginnt die Körpertemperatur allmählich zu sinken und erreicht ihren niedrigsten Wert bei alten Menschen.

\section{Axillare Grenzwerte}

$\begin{array}{ll}\text { Subfebril } & 36,9-37,4^{\circ} \mathrm{C} \\ \text { Fieber } & 37,6-39,5^{\circ} \mathrm{C} \\ \text { Hohes Fieber } & 39,5-40,5^{\circ} \mathrm{C} \\ \text { Sehr hohes Fieber } & \text { Über } 40,5^{\circ} \mathrm{C} \\ \text { Hyperpyrexie, Hyperthermie } & \text { Über } 41^{\circ} \mathrm{C}\end{array}$

\subsubsection{Subjektives und objektives Befinden}

Jeder erfahrene Arzt weiß, dass »Fieberfall nicht gleich Fieberfall« ist:

- So gibt es Fälle mit hohem UF, ohne dass die Patienten über ein schweres Krankheitsgefühl berichten. Dagegen können - z. B. bei tonsillären Anginen - nur mittelhohe Temperaturen gemessen werden; aber dennoch erscheint der Patient subjektiv und objektiv schwer krank. 
- Hohes Fieber bei Säuglingen und Kleinkindern beunruhigt die Mütter in besonderem Maße, besonders wenn kein Ausschlag zu sehen ist. Der Arzt dagegen fasst dies eher als Ausdruck einer guten Reaktionslage auf und kann die Mutter entsprechend beruhigen.

- Kleinstkinder halten Temperaturen um die $40^{\circ} \mathrm{C}$ nur einen, höchstens 2 Tage aus, da sie nicht genug trinken können, um den Wasserverlust auszugleichen. Zudem neigen sie bei hoher Körpertemperatur eher zu Fieberkrämpfen.

Besonders herumtollende Kinder (»Spielfieber») fühlen sich manchmal »heiß« an. Solche Temperaturerhöhungen nach motorischer Aktivität im Vorschul- und Schulkindalter sind bis $38,5^{\circ} \mathrm{C}$ durchaus in der Norm und sinken nach einer $0,5^{-1} \mathrm{~h}$ Ruhe wieder ab. Daher sollte bei Kindern erst $\mathrm{ab} 39^{\circ} \mathrm{C}$ von "Fieber « gesprochen werden. Auch Erwachsene zeigen nach sportlicher Betätigung ein gleiches Verhalten.

- Wenn dagegen ein alter Mensch mäßiges Fieber hat, halten die Angehörigen diesen Befund zunächst nicht im selben Maße für Besorgnis erregend. Erfahrene Ärzte wissen, dass hier oft der Schein trügt.

- Alten Menschen können bei Fieber Tachykardien, kardiale Erkrankungen bis hin zur Insuffizienz oder einer $\mathrm{O}_{2}$-Unterversorgung drohen. In diesen Fällen muss das Fieber mit einem Antipyretikum gesenkt werden.

\subsubsection{Wochenlange Temperaturerhöhungen}

Das »normale«, flüchtige UF ist $\mathrm{zu}$ unterscheiden vom (seltenen) wochenlang andauernden UF.

Solche ungeklärten Temperaturerhöhungen über mindestens 14 Tage hinweg treten in der Allgemeinpraxis gerade noch an der Grenze der regelmäßigen Häufigkeiten auf (-Tabelle A1), d. h. sie sind die Ausnahme von der Regel. Das »normale « UF klingt in 2-5 Tagen spurlos ab. Eine Dauer von 1 oder gar 2 Wochen ist die Ausnahme.

\subsubsection{Nicht mehr uncharakteristisch}

Nur in 1 von 4 Fieberfällen finden sich Krankheitszeichen, die eine Charakterisierung gestatten. Die große Mehrheit wird von fieberhaft verlaufenden Gesundheitsstörungen gebildet, die näher charakterisierende Zeichen und Befunde vermissen lassen.
Fieberhafte Erkrankungen sind per definitionem nicht mehr "uncharakteristisch «, wenn sich bei der Untersuchung z. B. ein Erysipel oder eine Thrombophlebitis als "charakteristische« Symptome aufdecken lassen. Dasselbe gilt, wenn sich vergrößerte Tonsillen mit eitrigen Belägen und ggf. mit angulären Lymphknoten finden.

In diesen Fällen ist also nicht UF zu klassifizieren, sondern das entsprechende Krankheitsbild (Erysipel, Thrombophlebitis, Tonsillitis o. Ä. - vgl. - Fallbeispiel, S. 20f).

Ebenso wenig "uncharakteristisch« ist Fieber dann, wenn z. B. bei liegendem Dauerkatheter ein Fieberschub auftritt (»iatrogenes Fieber«). Hier wird klarerweise - bei entsprechendem Laborbefund - eine Zystopyelitis (C) klassifiziert. Ähnliche Klassifikationsüberlegungen gelten, wenn eine Impfung vorausgegangen ist (Impffieber).

\subsubsection{Qualitätskontrolle am Beispiel des Fieber-Programms}

Die allermeisten UFs in der Allgemeinpraxis heilen nach einigen Tagen komplikationslos ab.

Haben die ersten Angaben des Patienten über die Beschwerden und Krankheitszeichen und hat die epidemiologische Situation bzw. der »erste Blick« des Arztes nicht in eine bestimmte Richtung (z. B. Masern, Stirnhöhlenoder Lungenentzündung, Pyelonephritis, Hepatitis) gelenkt, so kommt die standardisierte Diagnostik ( $\triangleright$ Checkliste Nr. 1,»Fieber-Programm«; $\bullet$ Übersicht 1.1) zum Tragen. Die Hauptzielrichtungen des Programms sind in • Abb. 1.3 dargestellt.

Diese Checkliste dient der systematischen Suche nach wichtigen "versteckten" Symptomen. Bei ausschließlich intuitivem Vorgehen lässt sich nicht die gleiche Verlässlichkeit erreichen (Braun 1988a).

Die große Stärke des Arbeitens mit diagnostischen Programmen liegt darin, dass alle wichtigen AGVs berücksichtigt werden.

Das programmierte Untersuchen beim UF zwingt den Arzt beispielsweise dazu, die Beweglichkeit des Nackens zu überprüfen, auch wenn zunächst nichts für eine Meningitis spricht. Es zwingt, die Lunge abzuhorchen, auch wenn nicht der geringste Verdacht auf eine Pneumonie besteht. Ebenso schreibt das Programm konsequent vor, die abdominelle Region zu palpieren, auch wenn nicht der mindeste Hinweis auf eine intraabdominelle Affektion (etwa auf eine Wurmfortsatzentzündung) besteht. 
Übersicht 1.1

\section{Checkliste Nr. 1 »Fieber-Programm«. (Braun u. Mader 2003)}

- Für uncharakteristische Fieberfälle und deren fieberfreie Varianten (afebrile Allgemeinreaktion)

(Fieber-Programm)

Subjektiv

\section{Epidemie}

Erster Eindruck (leicht/schwer krank)

Krank (Bettruhe) seit

Gleich/besser/schlechter

Schon mal gehabt

Fieberhöhe (axillar, rektal, Ohr, oral, geschätzt)

Fieberdauer/Fieberschübe

Mattigkeit/Appetitlosigkeit/Schlafstörungen

Frösteln/Schweiße

Nasenatmung/Atemnot

Ausschlag

Aktuelle Operation/Implantation von Fremdmaterial

Schnupfen/Niesen/Husten/Auswurf (klar/gelb/blutig)

Halsschmerzen/Heiserkeit

Kopf-, Ohrenschmerzen

Stamm-/Waden-/Glieder-/Gelenk-/Nackenschmerzen/ sonstige Schmerzen (z. B. Bauch/Mutterbrust)

Übelkeit/Brechreiz/ Erbrechen

Durchfall/Verstopfung

Pollakisurie/Algurie

Menstruelle Anomalien

Gewichtabnahme

Tropenreise/HIV-Möglichkeit

Genuss von roher Milch (Listeriose!)/Besonderes gegessen

Katze gekratzt (Felinose!)/Zecken-/Kanülenstich/Vogelhaltung

Berufliche Exposition

Ängste (Furcht vor)

Vermutete Ursache

Selbstbehandlung/ärztliche Anbehandlung

Sonst noch
Objektiv

Inspektion Körper/Beine (z. B. Erysipel!)

Nasensekretion (Spekulum)

Nasennebenhöhlen druckschmerzhaft

Gehörgang/Trommelfell (Otoskopie Kleinkind)

Mund/Rachen

Kopfbeugung frei

Halslymphknoten

Lungenauskultation/-perkussion

Herzauskultation

Abdomen palpatorisch

Nieren klopfempfindlich

Blutdruck/Puls

Labortests (Urinstatus)

Laborserologie (HIV)

Sonst auffällig

Beratungsergebnis

Maßnahmen 


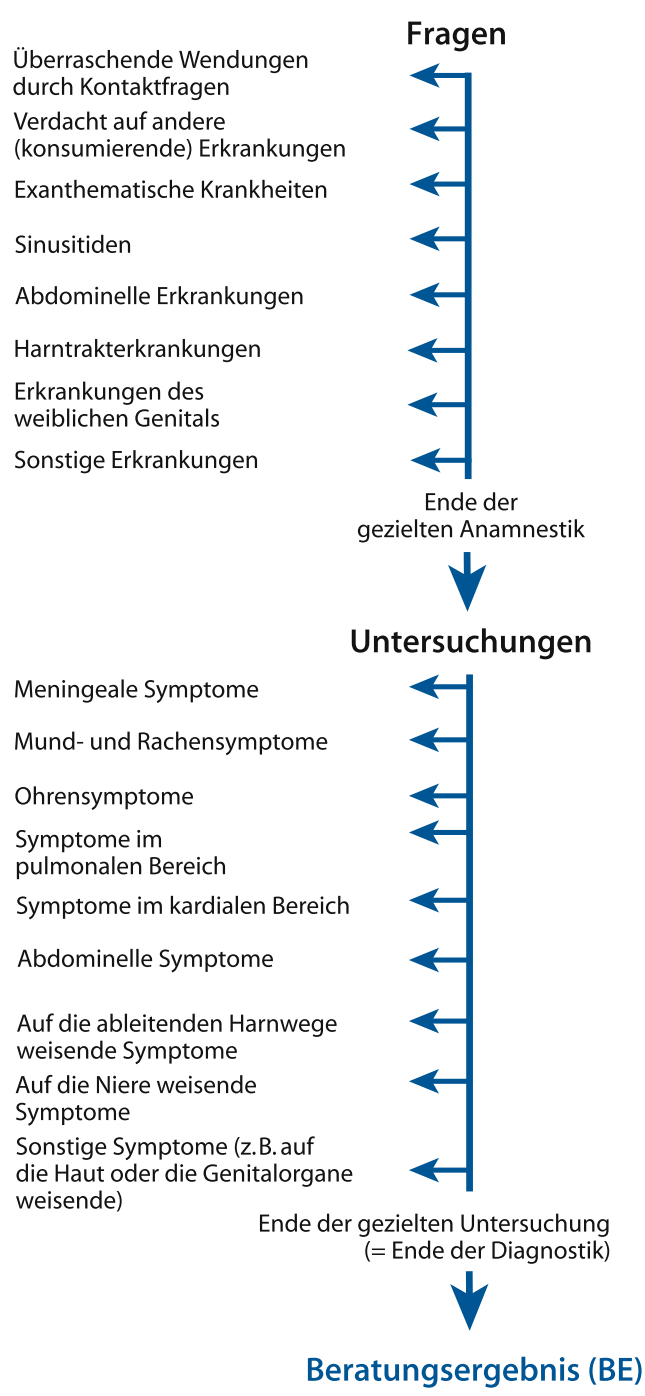

- Abb. 1.3. Die Hauptzielrichtungen der allgemeinärztlichen programmierten Diagnostik bei uncharakteristischem Fieber (UF). (Braun 1986)

Danninger deckte unter 200 standardisiert untersuchten Fällen von UF 11-mal unerwartete Erkrankungen oder Symptome einer Erkrankung auf; hierdurch kam eine diagnostische Weichenstellung zustande (• Tabelle 1.5).

(? Die programmierte Diagnostik führt im Übrigen nicht automatisch zu einer Diagnose (D). Die meisten Fälle, die sich uncharakteristisch präsentieren, bleiben auch nach dem Einsatz einer solchen Checkliste uncharakteristisch.
Der Arzt darf jedoch beruhigt sein, nichts Machbares unterlassen zu haben. Die Anwendung der programmierten Diagnostik stellt somit einen wichtigen Beitrag zur Qualitätskontrolle und zur Qualitätssicherung in der Allgemeinmedizin dar (• Abb. A4, S. 25).

\subsubsection{Management}

Für das Management beim UF ist die Feststellung wesentlich, ob das Fieber weniger oder länger als 6 Tage besteht.

Unter 7 Tagen sollte auch beim leicht kranken Patienten routinemäßig nach $\triangleright$ Checkliste Nr. 1 (»Fieber-Programm«; Übersicht 1.1) vorgegangen werden. Der Zeitaufwand für die komplette programmierte Diagnostik liegt bei durchschnittlich 4,5 min (2 min Befragung, 2,5 min Untersuchung; Chung 1986).

Ab dem 7.Tag ist bei UF oder AFAR, d.h. bei fieberfreien sonst gleichartigen Beschwerdebildern, außer der programmierten Diagnostik sofort die Röntgenuntersuchung der Thoraxorgane fällig, ebenso die Untersuchung der Blutkörperchensenkung (BKS), des C-reaktiven Proteins (CRP), der Leukozyten und des Urins; spätestens nach weiteren 24-48 h Temperaturerhöhung ist spezialistischer Rat zu holen, selbst bei nur geringem Krankheitsgefühl und trotz guten Aussehens. Je schwerer der Verlauf und je mitgenommener der Kranke ist, um so früher wird eingewiesen.

Bei wochenlang anhaltendem Fieber unbekannten Ursprungs sollte $u$. a. gedacht werden an:

- virale (»human immunodeficiency virus«, HIV) oder spezifische (z. B. tuberkulöse) Infektionen,

- endogene Toxine,

- exogene Gifte (Metallfieber, drugfever«),

- Tumoren,

- zentrales Fieber,

- hormonelle Einflüsse (z. B. Hyperthyreose),

- Kollagenosen,

- neurovegetative Einflüsse etc.

Es gibt bekanntlich auch - trotz größtem diagnostischen Aufwand - unaufklärbare Zustände.

Der Laie ist darauf bedacht, Fieber mit allen Mitteln rasch zu senken. Dabei geht er von der Überlegung aus, die Höhe des Fiebers stehe in direktem Zusammenhang mit der Schwere der Erkrankung. 


\begin{tabular}{|c|c|c|c|c|c|}
\hline Fall & Alter & Geschlecht & Beratungsursache & Beratungsergebnis & $\begin{array}{l}\text { Durch die programmierte } \\
\text { Diagnostik neu aufgedeckte Erkrankung }\end{array}$ \\
\hline 1 & $49 \mathrm{~J}$. & Männlich & Fieber, Husten & UF & Leistenhernie \\
\hline 2 & $66 \mathrm{~J}$. & Weiblich & Verkühlung & AFAR & Subikterus \\
\hline 3 & $29 \mathrm{~J}$. & Männlich & Verkühlung & Husten & Perforiertes Trommelfell \\
\hline 4 & $36 \mathrm{~J}$. & Männlich & Verkühlung & Bronchitis & Hämorrhagische Zystitis \\
\hline 5 & $20 \mathrm{~J}$. & Weiblich & Ohrenbeschwerden, Verkühlung & AFAR & Systolikum \\
\hline 6 & $6 \mathrm{M}$ & Männlich & Husten & Husten, Mesotitis & Anämie \\
\hline 7 & $27 \mathrm{~J}$. & Weiblich & Halsschmerzen, Husten & Pharyngitis & Zystitis \\
\hline 8 & $7 \mathrm{~J}$. & Weiblich & Fieber & UF, Bronchitis & Mikrohämaturie \\
\hline 9 & $55 \mathrm{~J}$. & Männlich & Fieber & UF & Albuminurie \\
\hline 10 & $42 \mathrm{~J}$. & Weiblich & Fieber, Brechreiz & UF & Diabetes mellitus \\
\hline 11 & $60 \mathrm{~J}$. & Männlich & $\begin{array}{l}\text { Reduzierter Allgemeinzustand, } \\
\text { Erbrechen }\end{array}$ & Depression & Depression \\
\hline
\end{tabular}

Die sofortige Anwendung von (Breitspektrum-) Antibiotika beim UF-Anfall kann gefährliche Verläufe verschleiern (etwa einen paranephritischen Abszess) oder die weitere Diagnostik erschweren (z. B. Kulturen "gehen nicht mehr an«).

Die vielfach in der Literatur als typisch beschriebenen Fieberverlaufskurven (z. B. bei Masern oder Sepsis) sieht man in der Praxis wegen der meist frühzeitig einsetzenden Therapie nur noch selten und im Übrigen oft erst im nachhinein.

Der Allgemeinarzt kann akute unklare Fälle durchaus einige Tage lang in geteilter Verantwortung mit dem Patienten abwartend offen lassen, bis es keinerlei Zweifel mehr am Ausbleiben einer Wendung zum Besseren gibt.

Der Patient und die Pflegepersonen sollen zu sorgfältiger Beobachtung angehalten werden. Sie sind zu instruieren, regelmäßig und exakt die Körpertemperatur zu messen. Die Führung einer Fieberkurve wird dabei auch für den zu Hause betreuten Patienten empfohlen, nicht zuletzt aus Gründen der Objektivierung, aber auch zur Information und aus psychologischen Gründen.

Beim UF soll der Arzt auf engen Kontakt mit der Patientenfamilie bedacht sein. Empfehlenswert sind telefonische Berichte in 12- bis 24-stündigen Abständen oder ein Hausbesuch zur Kontrolle. Jede Verschlechterung oder das Auftreten neuer, auffallender Symptome (z. B. Erbrechen), ist dem behandelnden Arzt unverzüglich bekannt- zugeben. Unter solchen Umständen kann der Arzt seiner Verantwortung bestens gerecht werden.

\subsection{Afebrile Allgemeinreaktion (AFAR)}

Die AFAR ist die afebrile Variante des UF.

In der allgemeinmedizinischen Fachsprache bedeutet AFAR:

- Allgemeinsymptome und/oder Lokalsymptome.

Bei der Untersuchung lassen sich keine charakteristischen Krankheitszeichen und Befunde erheben.

Die Häufigkeit der AFAR ist den • Tabellen 1.1 und 1.2 (S. 31 und 32) zu entnehmen.

Existieren bei uncharakteristischen Erkrankungsfällen der genannten Art zwar örtliche und Allgemeinerscheinungen, fehlt jedoch die erhöhte Körpertemperatur (= AFAR), so ist die $\triangleright$ Checkliste Nr. 1 (»Fieber-Programm «; - Übersicht 1.1) ebenso indiziert wie beim UF.

\subsection{Afebriler Husten}

Afebriler Husten als alleiniges Symptom (A), also ohne Fieber und ohne Allgemeinerscheinungen, mit und ohne Auswurf, zählt zu den häufigsten BEs in der Allgemeinme- 
dizin; besonders die Altersgruppen 0-14 Jahre sind davon betroffen.

\subsubsection{Intuitive primäre Diagnostik}

Hustet ein Kind seit wenigen Tagen, bestehen keine Allgemeinerscheinungen (also kein Fieber, keine Abgeschlagenheit, keine Appetitlosigkeit, kein krankes Aussehen) und hat die Mutter beispielsweise nur den Wunsch nach Verordnung eines Hustensaftes, dann darf der Arzt dieses Ansinnen nicht sogleich erfüllen. Seine geringste Pflicht in einem solchen Fall ist,

- nach einem vorher abgelaufenen Fieber und/oder anderen Allgemeinerscheinungen zu fragen,

- nach einer Fremdkörperaspiration zu fragen,

- den Rachen zu inspizieren,

- die Lunge abzuhorchen.

Davon abgesehen muss eine entsprechende Seuchenlage gegeben sein, d. h. keine Masern, kein Keuchhusten usw.

Nach der intuitiven negativen primären Diagnostik können ein Rezept verschrieben sowie Mutter und Kind verabschiedet werden. Die Mutter ist anzuhalten, bei einer Verschlechterung oder bei unveränderter Fortdauer des Hustens über 1 Woche mit dem Kind erneut zum Arzt zu kommen.

\subsubsection{Verschlimmerung}

Beim akuten uncharakteristischen Husten genügt es, intuitiv vorzugehen und sich auf einige gezielte Fragen und auf die lokale Untersuchung zu beschränken.

Dauert der Husten 1 oder 2 Wochen, so muss grundsätzlich programmiert untersucht werden (»Husten-Programm ", > Checkliste Nr. $2^{1}$ ). Im Vordergrund stehen hier bei Kindern die Exklusion einer Pneumonie, bei Erwachsenen außerdem die Exklusion eines Malignoms und einer Tuberkulose.

Wird der Husten stärker, so hängt es vom Allgemeinzustand des Patienten ab, ob man ihn sogleich oder am 10.-14. Krankheitstag zur Röntgenaufnahme der Thorax-

\footnotetext{
${ }^{1}$ Alle in diesem Buch genannten Checklisten/Programme sind enthalten in Braun RN, Mader FH (2003) Programmierte Diagnostik in der Allgemeinmedizin. 82 Checklisten für Anamnese und Untersuchung. 4. Aufl. Springer, Berlin Heidelberg New York.
}

organe überweist. Besteht der Husten bereits seit 2 Wochen, so muss sofort eine Röntgenuntersuchung erfolgen.

Bei Patienten mit über 8 Wochen persistierendem Husten sollte zusätzlich zur Röntgenaufnahme eine Abklärung in Form einer Lungenfunktionsdiagnostik, ggf. Bodyplethysmographie (Fluss-Volumen-Kurve, Bronchodilatation oder unspezifische Provokation) und Allergiediagnostik [PrickTest, Gesamt-Immunglobulin- (Ig)E, Radioallergosorbenttest (RAST)] erfolgen sowie u.U. eine Computertomographie des Thorax, eine Bronchoskopie und eine 24-h-pH-Metrie (gastroösophagealer Reflux!) durchgeführt werden.

Bei älteren Menschen können durch eine solche Stufendiagnostik Malignome (meistens leider unabwendbar gefährliche Verläufe), bei Patienten mittleren Alters etwa Tuberkulose, bei Kindern Pneumonien (d.h. potenziell gefährliche Verläufe) entdeckt werden. Solche Ergebnisse sind im Praxisalltag jedoch - von Pneumonien abgesehen - Raritäten.

Bei Fieber und/oder Husten darf sich der Allgemeinarzt nicht durch die überwältigende Dominanz des Banalen dazu verleiten lassen, nur oberflächlich oder gar nicht zu untersuchen. Vielmehr hat er stets ein möglichst volles Untersuchungsprogramm allgemeinmedizinischer Art abzuwickeln.

\subsubsection{Symptomgruppe "Bronchitis»}

Bronchitis bedeutet in der Nomenklatur der berufstheoretischen Fachsprache keine exakt definierte Krankheit, sondern stellt eine Klassifizierung von mehreren Symptomen (B) dar.

$\mathrm{Zu}$ dieser Symptomgruppe zählen u. a. die bei der Auskultation zu findenden beidseitigen, nichtklingenden Rasselgeräusche bei normalem Exspirium, die sich nicht weghusten lassen.

Das $\mathrm{BE} »$ Bronchitis« ist von der »Bronchitis asthmatica" (ausführlich in $>$ Abschn. B5.11) dadurch abzugrenzen, dass bei der asthmatischen Bronchitis ein verlängertes Exspirium mit Giemen, Pfeifen etc. gefunden wird.

Die Erkrankung dauert in der Regel wenige Tage oder Wochen und ist im Gegensatz zur Bronchitis asthmatica nicht durch rezidivierende Atembeschwerden oder permanente Überempfindlichkeit des Bronchialsystems charakterisiert. 


\subsubsection{Keuchhusten}

Viele medizinische Laien, aber auch manche Ärzte sind der Ansicht, dass Keuchhusten, der bereits 1640 erstmals als "Pertussis« beschrieben wurde, eine Erkrankung des Kindesalters sei. Tatsache jedoch ist, dass heute vor allem durch den Impfrückgang eine zunehmend größere Anzahl von Erwachsenen von Bordetella pertussis befallen ist.

Das Immunsystem von Säuglingen ist gegen B. pertussis wehrlos. So können Jugendliche oder Erwachsene, die unter lang anhaltendem Husten leiden, Babys durch Anhusten infizieren (»Tröpfcheninfektion«). Dasselbe gilt auch für die Infektion mit den eher seltenen B.-parapertussis-Bakterien.

Bei Kindern ist die Infektion mit B. pertussis charakterisiert durch anfallsweisen Husten, »juchzendes« Einziehen der Luft (»whoop«), durch anschließendes Erbrechen, Atemnot oder Gesichtszyanose (paroxysmales Stadium oder Stadium convulsivum). Oft kann es noch monatelang (auch trotz Therapie) zu gelegentlichen Hustenattacken kommen (Stadium decrementi).

Besonders gefürchtet sind bei Säuglingen die folgenden Komplikationen:

- Pneumonie (22\%),

- Apnoe und hypoxisch bedingte Enzephalopathie mit Krämpfen (11\%),

- kardiale Schäden (3,5\%).

D Pertussis beginnt als uncharakteristischer Husten und/ohne Fieber (katharralisches Stadium). Zahlreiche Erreger, u. a. Adenoviren, erzeugen ein pertussisähnliches Bild.

Die World Health Organization (WHO) definiert Pertussis als "Husten von mindestens 3-wöchiger Dauer mit paroxysmalen Hustenanfällen«. Dabei ist zu beachten, dass ein Viertel der Keuchhustenfälle durchaus kürzer als 3 Wochen dauert.

Im (»typischen «) Stadium convulsivum weist das Differenzialblutbild eine ausgeprägte Leukozytose (20.000$30.000 / \mu \mathrm{l})$ sowie eine Lymphozytose (60-80\%) auf. Der Erreger kann im Nasopharyngealsekret oder mithilfe der Polymerase-Kettenreaktion ("polymerase chain reaction«, PCR) nachgewiesen werden. Bei älteren Kindern oder Erwachsenen dagegen ist die Serologie sensitiver als der Erregernachweis. Die Antikörperbestimmung erfolgt in der 2.-4. Woche. Im frühen Stadium convulsivum ist die Serologie noch negativ.
Die Therapie der Wahl ist Erythromycin für 2 Wochen, aber auch neuere Makrolidantibiotika sind wirksam. Empfängliche Kontaktpersonen des Indexfalls (wie Familienmitglieder) sollten für 14 Tage eine Chemoprophylaxe mit einem Makrolid erhalten. Nicht vollständig geimpfte Kinder profitieren von einer Boosterimpfung.

D Die Impfung wegen Pertussis mit dem durchwegs verträglichen azellulären Impfstoff ist für alle Altersgruppen sinnvoll. Kein lebenslanger Schutz, daher Auffrischungsimpfungen erforderlich!

\subsection{Halsschmerzen}

Afebrile Halsschmerzen (ohne örtliche Entzündungserscheinungen) sind ähnlich wie die afebrile Pharyngitis (d.h. Halsschmerzen mit örtlichen Entzündungen) von Druck-, evtl. von Trockenheitsgefühl im Hals, auch von Schluckbeschwerden begleitet.

Das Globusgefühl wird vom Patienten tiefer im Hals angegeben.

\subsubsection{Tonsillitis}

Die akute Mandelentzündung stellt den Hausarzt bezüglich ihrer ätiologischen Zuordnung immer wieder vor Probleme:

Bei allen Halsschmerzen mit und ohne Fieber können Mandelbeläge auftreten. Dabei ist es dem Arzt nicht möglich, vom Aspekt her zu entscheiden, welche Erreger (Bakterien, Viren, Pilze) zugrunde liegen.

(. Eine »eitrige Tonsillitis" gibt es nicht. Der Begriff suggeriert fälschlicherweise, dass weiße (oder gelbliche) Beläge auf den Tonsillen Beweis für eine »pyogene Infektion « seien, und dass deswegen eine antibiotische Therapie indiziert ist.

Finden sich kleine Bläschen auf den Tonsillen, ist eine akute Infektion durch Herpes- oder Coxsackieviren wahrscheinlich.

D Bei Kleinkindern können Bauchschmerzen mit Übelkeit und Erbrechen im Vordergrund stehen.

Die Tonsillitis acuta muss abgegrenzt werden gegen:

- UF (Tonsillitis wenig oder kaum ausgeprägt);

- Pharyngitis (Tonsillitis fehlt; keine weiteren Symptome); 
- Scharlach (typisches Exanthem, Himbeerzunge; später Schuppung an Handflächen und Fußsohlen; - Abschn. A1.3; Abschn. B12.5);

- Mononucleosis infectiosa ( A Abschn. B1.5.2);

- Halsschmerzen ( Abschn. B1.5);

- Angina Plaut-Vincent.

Die Tonsillitis chronica verursacht häufig typischerweise Schmerzen, die in das Ohr (die Ohren) ausstrahlen. Die Indikation zur Tonsillektomie sollte im Erwachsenenalter besonders streng gestellt werden.

Der Peritonsillarabszess ist die häufigste Komplikation der entzündlichen Mandelerkrankungen. Er tritt nach symptomfreiem Intervall auf. In der Allgemeinpraxis sieht jedoch der Arzt nur noch den Abszess, ohne zuvor eine Tonsillitis behandelt zu haben.

(. Bei protrahiertem Verlauf einer eitrigen Angina tonsillaris ist an den AGV eines Peritonsillarabszesses zu denken.

Die Ulzerationen bei der Plaut-Vincent-Angina sind in der Regel wenig eindrucksvoll, die grau-grünlichen Beläge dagegen sind auffallend.

? Wenn bei einer Plaut-Vincent-Angina das Ulkus 2-3 Wochen persistiert, muss ein Karzinom ausgeschlossen werden.

Eine spezifische Behandlung der Mandelentzündung ist - außer bei A-Streptokokken-Tonsillitis - nicht erforderlich. Man gibt heute Penicillin V 100.000 IE/kg KG in 2 (bis 3) Einzeldosen für 10 Tage. Ob durch eine kürzere Therapiedauer die nichtsuppurativen Komplikationen (langfristig) zunehmen, ist nicht geklärt. Da innerhalb von $24 \mathrm{~h}$ nach Therapiebeginn eine Besserung der Symptomatik eintritt (ansonsten war die »Diagnose« falsch), geben die meisten Eltern ihren Kindern das Penicillin ohnehin nur für wenige Tage.

(. Die Häufigkeit des rheumatischen Fiebers lässt sich durch die Gabe von Penicillin reduzieren, die Häufigkeit der Glomerulonephritis jedoch nicht.

Für die streptokokkenbedingte Glomerulonephritis gibt es keine ursächlich wirksame Therapie. Es ist also völlig ausreichend, mit der vertieften Diagnostik so lange zu warten, bis entsprechende Symptome aufgetreten sind. Ein solches Vorgehen ist möglicherweise sinnvoller, als Eltern über Wochen und Monate hinweg darüber bangen zu lassen, ob ihr Kind tatsächlich eine Niereninsuffizienz entwickeln wird (Schmitt 2002).

(1)

Nach Penicillintherapie sind »Folgeabstriche« zum Nachweis von A-Streptokokken nicht indiziert.

\subsubsection{Infektiöse Mononukleose}

Bei sehr hohem Fieber im Kleinkindesalter sowie bei Jugendlichen (verbunden mit retronuchaler und/oder submandibulärer Lymphknotenvergrößerung sowie mit Halsweh und (vor allem bei Jugendlichen) Mandelentzündung mit auffallenden Belägen sollte der Hausarzt eine infektiöse Mononukleose (Pfeiffer-Drüsenfieber) in Betracht ziehen. Hinzu kann eine Vielzahl weiterer Symptome, wie Abgeschlagenheit, Kopf- und Gliederschmerzen, Ausschlag oder Leber- und Milzvergrößerung, kommen.

Als Erreger wurde 1964 das Epstein-Barr-Virus (EBV) bei Patienten mit Burkitt-Lymphom entdeckt. Es ist weit verbreitet und gehört neben Herpes simplex (HSV), Varicella-Zoster-Virus (VZV) und Zytomegalievirus (CMV) zur Gruppe der humanpathogenen (DNS-)Herpesviren.

Der erste Ansteckungsgipfel liegt zwischen dem 1. und 3. Lebensjahr mit häufig asymptomatischem oder subklinischem Verlauf. Zwischen dem 14. und dem 20. Lebensjahr ist ein zweiter Ansteckungsgipfel mit dem Krankheitsbild der infektiösen Mononukleose (»kissing disease«) bzw. eine Reaktivierung zu beobachten.

(I) Nach der ersten Infektion persisistiert das EBV lebenslang in B-Lymphozyten und Epithelzellen des Nasen-Rachen-Raumes. Somit bleibt jeder Infizierte ein Virusträger.

In seltenen Fällen kann es zu schwer wiegenden Komplikationen, wie Pneumonie, Meningoenzephalitis, Myokarditis oder Milzruptur, kommen. Charakteristisch im Differenzialblutbild sind Leukozytose und monozytoide Lymphozyten (Pfeiffer-Zellen, »buntes Bild «).

Darüber hinaus steht dem Hausarzt heute eine leistungsfähige Diagnostik im Speziallabor zur Verfügung (• Tabelle 1.6). Der Nachweis heterophiler Antikörper im Paul-Bunnell-Test bei einer frischen infektiösen Mononukleose hat aufgrund seiner vor allem im Kindesalter geringen Sensivität von $50 \%$ an Bedeutung verloren. 


\begin{tabular}{|c|c|c|}
\hline \multicolumn{2}{|c|}{$\begin{array}{l}\text { Keine EBV-spezifischen Reaktionen bei der IgG-, } \\
\text { IgM- und IgA-Bestimmung }\end{array}$} & \multirow{2}{*}{$\begin{array}{l}\text { EBV-negativ } \\
\text { Erstinfektion - Infektiöse Mononukleose }\end{array}$} \\
\hline $\lg G$ & EBNA-1 und VCA p18 negativ, VCA p23 und EA möglich & \\
\hline $\lg M$ & EA positiv;VCA p23 möglich & \\
\hline $\lg G$ & $\begin{array}{l}\text { EBNA-1 und/oder VCA p18 positiv, VCA p23 häufig positiv, } \\
\text { schwache EA-Titer möglich; in 5\% der Fälle auch Anti-EBNA-1- } \\
\text { Verlust möglich }\end{array}$ & Abgelaufene Infektion \\
\hline $\lg M$ & Keine Reaktivität & \\
\hline $\lg G$ & Starke Reaktivität mit allen Antigenen einschließlich mit EA & $\begin{array}{l}\text { Sekundäre Reaktivierung (EBV nicht in die Symptomatik } \\
\text { einbezogen) }\end{array}$ \\
\hline $\lg A$ & Keine Reaktivität gegen EA und/oder VCA & \\
\hline $\lg G$ & $\begin{array}{l}\text { Starke Reaktion mit allen Antigenen, in Ausnahmen } \\
\text { Anti-EBNA-1-Verlust }\end{array}$ & Reaktivierung \\
\hline $\lg M$ & Schwache Reaktivität mit EA und VCA möglich & \\
\hline $\lg A$ & Deutliche Reaktivität mit EA und/oder VCA & \\
\hline \multicolumn{2}{|c|}{ Serologisches Bild wie Reaktivierung (hohe IgA-Titer!) } & Nasopharynxkarzinom und EBV-assoziierte Lymphome \\
\hline
\end{tabular}

\subsection{Heiserkeit}

Die isolierte Heiserkeit (A) ergibt sich als BE vorwiegend bei jüngeren Erwachsenen. Der Patient kommt schon nach ganz wenigen Tagen der Erkrankung zum Arzt. Die Stimme ist heiser, die Racheninspektion unauffällig.

Dauert die Heiserkeit länger als 1 Woche oder handelt es sich um ältere Menschen, so ist die Laryngoskopie obligat. Nach spätestens 2 Wochen ist die Untersuchung durch einen Spezialisten indiziert.

Im Allgemeinen klingt eine Heiserkeit mit und ohne verifizierter Laryngitis im Verlauf von 2-3 Wochen wieder ab.

Ältere, innerhalb kurzer Zeit heiser gewordene Patienten, ob Raucher oder nicht, die weder UF noch eine AFAR noch Luftwegekatarrhe ohne Allgemeinerscheinungen aufweisen, sind sofort in den spezialistischen Bereich zur Diagnostik zu $\nabla$ überweisen. Dasselbe gilt für Kinder und sonstige leicht kranke Erwachsene, bei denen nach 1 Woche Beobachtung eine Besserung der Beschwerde ausbleibt.

\subsection{Schnupfen}

Die Mehrzahl der Patienten mit isoliertem Schnupfen (afebrile Rhinitis; A) sucht den Arzt nicht auf. Der Nasenfluss wird vom Patienten eher als störend denn als gefährlich empfunden und mit Hausmitteln selbst behandelt.

Die tief schürfende Abklärung des Schnupfens ist nur bei Verdacht auf allergiebedingten Schnupfen oder bei Verdacht auf Fremdkörper indiziert.

Für das Kleinkind und noch viel mehr für den Säugling stellt der akute Schnupfen eine oft ernste Beeinträchtigung des Allgemeinbefindens dar; deshalb kann auch von einer "Schnupfenkrankheit" gesprochen werden. Die jährliche Inzidenz liegt zwischen 2 und 20 Erkrankungen; kleinere Kinder erkranken häufiger als ältere. 


\subsubsection{Inanspruchnahme des Arztes}

Eine Stichprobe im unausgelesenen Krankengut einer Allgemeinpraxis (223 Patienten) ergab, dass 85\% der Befragten ab und zu Schnupfen haben; nur 15\% litten nie an Schnupfen. Von denen, die angaben, ab und zu unter Schnupfen zu leiden, hatten sich $52 \%$ selber Medikamente besorgt; $31,6 \%$ fanden es nicht notwendig, den Schnupfen zu behandeln, nur $16 \%$ gaben den Schnupfen als alleinigen Grund für einen früheren Arztbesuch an. Aus dieser Befragung geht erwartungsgemäß hervor, dass die Mehrzahl der Patienten ab und zu Schnupfen hat, aber deshalb nur selten zum Arzt geht.

Bei 370 (mithilfe des »Fieber-Programms «, $\triangleright$ Checkliste Nr. 1; Übersicht 1.1) untersuchten Patienten wurde in 201 Fällen (54\%) UF und bei 127 Patienten (34\%) eine AFAR klassifiziert. Bei $11 \%$ der Fälle ließ sich nicht erheben, ob die Temperatur gemessen wurde. In beiden Gruppen litten rund $70 \%$ der Patienten an Schnupfen. Bei über zwei Drittel der Patienten - ob mit oder ohne Fieber - war also Schnupfen vorhanden (Landolt-Theus 1986).

Ein Teil der Patienten mit Schnupfen glaubt, die Ursache des Schnupfens zu kennen. Diese Erkrankten kommen zum Arzt und geben von vornherein an, dass sie unter "Heuschnupfen « oder unter einem allergischen Schnupfen leiden. Sie wollen entweder die erprobte Therapie fortsetzen oder wünschen eine Abklärung der Ursache.

\subsubsection{Verlauf}

Aus der Verlaufsdauer lässt sich manchmal auch auf die Art des Schnupfens schließen:

- allergisch: Schnupfendauer entsprechend der Dauer des Pollenflugs,

- nichtallergisch: in der Regel Abheilung innerhalb weniger Tage,

- chronisch: praktisch dauernd verstopfte oder rinnende Nase.

\subsection{Kombinierte Luftwegekatarrhe}

Die kombinierten Luftwegekatarrhe grenzen sich vom UF und der AFAR durch fehlende Allgemeinerscheinungen ab.

Der betroffene Patient klagt in solchen Fällen über Schnupfen und/oder Halsschmerzen und/oder Heiserkeit und/oder Husten ohne Allgemeinerscheinungen.
»Catarrhal child « nennt man im englischsprachigen Raum einen "ewig" verschnupften und hustenden, gelegentlich fiebernden kleinen Patienten. Früher oder später erhebt sich für die Eltern die Frage, ob es denn da wirklich keine Hilfe gibt. Zu diesem Zeitpunkt liegen schon verschiedene erfolglose therapeutische Bemühungen hinter ihnen.

Im spezialistischen Bereich gibt es z. B. die Bezeichnungen »Rhinobronchitis« oder»Tracheobronchitis«. Damit wird ausgedrückt, dass verschiedene "Etagen « des Luftwegeapparates (ggf. in wechselnder Intensität und Reihenfolge) betroffen sein können (»Etagenwechsel«).

\subsection{Kruppbilder}

Notfälle im Kehlkopfbereich, die mit inspiratorischer Atemnot einhergehen, sind in der Allgemeinpraxis von heute - im Unterschied zur Nachkriegszeit - nicht mehr regelmäßig häufig.

Aufgrund der gegenwärtigen epidemiologischen Situation ist der echte (diphtherische) Krupp eine extreme Rarität mit einem Vorkommen von weit seltener als 1:100.00o Praxisfällen.

Etwa 1-mal unter 10.00o Fällen wird der Allgemeinarzt mit der akuten stenosierenden (subglottischen) Laryngotracheitis (»Pseudokrupp «1) konfrontiert.

Sie tritt, wie das UF, jedoch mit Stridor als dominierendem Symptom, auf. Als Rarität kann es einen solchen Pseudokrupp auch bei Smog, Keuchhusten usw. geben.

Diese verschiedenen Bezeichnungen der Laryngotracheitis im Laufe der letzten 100 Jahre zeigen einmal mehr die Wechselhaftigkeit fachsprachlicher Bezeichnungen ( $\triangleright$ Abschn. B2.1.1, Abschn. B2.3.2 und Abschn. B5.11.1).

Beim Pseudokrupp kommt es unter mehr oder weniger schweren Allgemeinerscheinungen meist sehr rasch zu bellendem Husten mit Stridor und Heiserkeit bis hin zur Aphonie.

\footnotetext{
1 »Krupp« ist ein Symptom. Durch den Zusatz von »Pseudo« sollte zunächste eine unspezifische Laryngitis mit Krupperscheinungen vom »echten diphtherischen Krupp« abgegrenzt werden, so auch von Hennoch 1890. Von der ersten Hälfte des 20. Jh. an nannte man die akute stenosierende subglottische Laryngitis vielfach »Grippekrupp«, während die Bezeichnung »Pseudokrupp« für nächtliche Atembeschwerden mit kruppartigen Symptomen ohne erkennbare entzündliche Erscheinungen verwendet wurde. Für ähnliche Zustände taucht neuerdings, vorwiegend im amerikanischen Schrifttum, der wenig glückliche, ungenau definierte Begriff »spasmodic croup« auf.
} 


\begin{tabular}{|c|c|c|}
\hline & Laryngotracheitis & Epiglottitis \\
\hline Allgemeinzustand & Befriedigend & $\begin{array}{l}\text { Schwer krank } \\
\text { Toxisch } \\
\text { Schock }\end{array}$ \\
\hline Körpertemperatur & Muss nicht erhöht sein & In der Regel erhöht \\
\hline Begleitrhinitis & Häufig & Nein \\
\hline Inspiratorischer Stridor & Stets, laut hörbar & Nur ausnahmsweise \\
\hline Husten & Bellend (»Krupphusten«) & Selten \\
\hline $\begin{array}{l}\text { Hals- und Schluckschmerzen, } \\
\text { Speichelfluss }\end{array}$ & Nein & Häufig \\
\hline Stimme, Sprache & Heiser bis aphonisch & Leise, verhalten, kloßig, aber nicht heiser \\
\hline Erreger & Viren & Meist Haemophilus influenzae \\
\hline Allgemeine Maßnahmen & $\begin{array}{l}\text { Kühle, feuchte Luft (z. B. Badezimmer) } \\
\text { Herumtragen, Beruhigung }\end{array}$ & $\begin{array}{l}\text { Veranlassung der sofortigen stationären } \\
\text { Einweisung mit ärztlicher Begleitung }\end{array}$ \\
\hline $\begin{array}{l}\text { Erstbehandlung durch den } \\
\text { Allgemeinarzt }\end{array}$ & $\begin{array}{l}\text { Hochdosiert Kortikoide, rektal } 100 \mathrm{mg} \\
\text { Prednison, Wirkungseintritt nach 30-45 min, } \\
\text { Sedierung: Chloralhydrat Rektiole }\end{array}$ & Gabe von Kortikoiden wirkungslos \\
\hline
\end{tabular}

Das charakteristische Symptom (Stridor) tritt meist plötzlich, nachts oder in den frühen Morgenstunden auf; Rezidive kommen vor.

Die perakute (supraglottische) Epiglottitis tritt im Verhältnis zur akuten stenosierenden (subglottischen) Laryngotracheitis in jedem 10. Fall, also noch viel seltener, auf. Diese Erkrankung ist charakterisiert durch ihre Schwere, die den Patienten meist aus voller Gesundheit heraus trifft (•Tabelle 1.7). Die Epiglottitis ist in der Regel, die Laryngotracheitis in den meisten Fällen von Fieber begleitet. Die Sprache ist kloßig.

(1) Der Altersgipfel der seltenen Epiglottitis liegt gewöhnlich bei 3-5 Jahren. Diese "Kinderkrankheit" befällt jedoch auch Erwachsene. Achtung: hochakute Lebensgefahr! Sofortige stationäre Einweisung mit Notarzt unter Sauerstoffgabe! Cave: Sedierung und Racheninspektion bei fehlendem inspiratorischen Stridor (epiglottitisches Ödem!; Gerrish et al. 1987).
Bei Stridor im Rahmen eines akuten, sonst uncharakteristischen fieberhaften Geschehens empfiehlt sich die - Checkliste« Nr. 5 (»Pseudokrupp-Programm«).

\subsection{Fieberkrampf}

Fieberkrämpfe (Fraisen) treten als Anfälle bei überwiegend normal entwickelten Kindern im Säuglings- und Kleinkindesalter (zwischen 3 Monaten und 5 Jahren) auf. Sie sind in den letzten 50 Jahren seltener geworden, derzeit nicht mehr regelmäßig häufig.

Da diese Krämpfe nur bei Fieber (meist $<39^{\circ} \mathrm{C}$ ) vorkommen, sind sie mit epilepsiebedingten Anfällen nicht $\mathrm{zu}$ verwechseln. Eine familiäre Disposition wird diskutiert. Die Angehörigen rufen in höchster Besorgnis den Arzt; wenn er eintrifft, ist der Krampfanfall für gewöhnlich vorbei. Der Anfall dauert in der Regel 1-3 min. 
Der "einfache Fieberkrampf» ist charakterisiert u. a. durch die primär generalisierte Anfallsform und unterscheidet sich dadurch phänomenologisch nicht von einem Grand Mal bei Epilepsie. Postparoxysmal treten i. Allg. keine Paresen auf; die Anfälle ereignen sich 1-mal innerhalb von $24 \mathrm{~h}$ und insgesamt höchstens 4-mal.

Der »komplizierte Fieberkrampf« dauert länger als 20 min, oder es treten Anfallserien auf.

(. Fieberkrämpfe von über 10 bis 15 min Dauer sind Notfälle! Bei jedem Krampfanfall unter Fieber ist stets an eine entzündliche Erkrankung des Zentralnervensystems (ZNS) zu denken. Im Zweifelsfall Lumbalpunktion!

Seltene, offensichtlich bedrohliche Fälle sollten unverzüglich ins Krankenhaus eingewiesen werden. Ist ein solches Ereignis zum ersten Mal aufgetreten, so ist der Hausarzt ebenfalls gut beraten, die Verantwortung mit dem Spezialisten zu teilen (ambulant oder stationär), letztlich zur Beruhigung der Angehörigen. Ansonsten empfiehlt sich frühestens 8 Tage nach dem Krampfanfall die Ableitung eines Elektroenzephalogramms (EEG), da ein Übergang in ein zerebrales Anfallsleiden möglich ist.

Die Therapie besteht zunächst in:

- der Beruhigung der Angehörigen,

- der Gabe eines Sedativums (Diazepam 0,3-0,7 mg/ kg KG i.v., alternativ: Clonazepam (Rivotril o,o1o, $07 \mathrm{mg} / \mathrm{kg}$ KG i.v.),

- der Gabe von fiebersenkenden Mitteln.

Wie weit Antipyretika prophylaktisch in der Lage sind, einen Fieberkrampf zu verhindern, wird derzeit diskutiert.

Von besonderer Wichtigkeit bleiben jedoch für den Hausarzt die Aufklärung und die Führung der Angehörigen sowohl im Hinblick auf einen möglichen späteren erneuten Anfall, aber auch im Hinblick auf anstehende Impfungen, die prinzipiell ein sog. Impffieber hervorrufen können.

Das Risiko für ein Fieberkrampfrezidiv liegt durchschnittlich bei $30 \%$. Ein erhöhtes Risiko besteht dann, wenn eine Belastung der Elterngeneration und/oder der Geschwister mit Fieberkrämpfen vorliegt oder der erste Fieberkrampf während des 1. Lebensjahres aufgetreten ist. Die Prognose ist in der Regel günstig: Etwa 60\% der Fälle treten nach dem 5. Lebensjahr nicht mehr auf.

\section{Thematik \\ des Fachgesprächs}

\section{Aufgabe}

Besprechen Sie die in > Übersicht 1.2 aufgeführten BEs, die mit oder ohne Fieber einhergehen, anhand der nachfolgenden Fragen und der bei den entsprechenden BEs aufgeführten Zusatzfragen!

\section{Übersicht 1.2}

Beratungsergebnisse, die mit oder ohne Fieber einhergehen können

- Regelmäßig häufig in der Allgemeinmedizin

- UF

- Afebriler Husten

- AFAR

- Afebrile Luftwegekatarrhe (obere und/oder untere Luftwege)

- Afebrile Pharyngitis (= Halsschmerzen mit Entzündungszeichen)

- Angina tonsillaris

- Afebrile Rhinitis (Schnupfen)

- Afebrile Halsschmerzen, ohne Befund

- Afebrile Laryngitis (Heiserkeit)

- Zustand nach UF

- Afebrile Bronchitis

- Wochenlange Temperaturerhöhungen

- Nicht regelmäßig häufig (= unter 1:3.000 Fälle)

- Kruppbilder

- Fieberkrämpfe (Fraisen)

- Angina Plaut-Vincent

- Impffieber

- Mononukleose

\section{Fragen »uncharakteristisches Fieber (UF), afebrile Allgemeinreaktion (AFAR), Luftwegekatarrhe, Tonsillitis»}

1. Ungefähre Häufigkeit in der Allgemeinpraxis.

2. Überlegungen zu Klassifizierung und Diagnose.

3. Notwendigkeit und Dringlichkeit des Hausbesuches, Zumutbarkeit und Psychologie der Einbestellung des fiebernden Patienten in die Praxis.

4. Alter der Patienten. 
5. Subjektive Merkmale:

- erster Eindruck des Arztes (z. B. schwer krank),

- Fieberdauer,

- Art der Temperaturmessung (axillar, rektal, sublingual, Ohr),

- sonstige Allgemeinerscheinungen (z. B. Appetitlosigkeit, Schlaflosigkeit),

- Kontaktfragen (z. B. »schon gehabt?«),

- örtliche Symptome (z. B. Husten, Auswurf, Halsschmerzen, Gliederschmerzen),

- Mutmaßung des Patienten (z.B. Ansteckungsquelle),

- bisherige Therapie inklusive Selbstmedikation (z. B. Fieberzäpfchen, Wickel).

6. Objektive Merkmale:

- Fieberhöhe, Temperaturschwankungen,

- optimale körperliche Untersuchung (z. B. Kopfbeugung bei Sitzenden, Otoskopie bei Kindern, Palpation von regionalen Lymphknoten, Palpation des Abdomens),

- programmierte Diagnostik (S.37),

- Labordiagnostik (beim Allgemeinarzt, durch den Spezialisten),

- Einsatzzeitpunkt und Aussagekraft bildgebender Verfahren (z.B. Diaphanoskopie, Röntgenaufnahme).

7. Beispiele für Erreger und Auswahl der entsprechenden Antibiotika.

8. Abwartendes Offenlassen. (»Wie lange?«)

9. Überweisung zum Spezialisten, stationäre Einweisung.

10. Beispiele für atypische AGVs, »Fallstricke« (z. B. Appendizitis, Pneumonie).

11. Behandlung durch den Arzt:

- Empfehlungen an die Patienten/Eltern (z. B. Bettruhe, Diät, Arbeitsunfähigkeit, verstärkte Flüssigkeitszufuhr),

- lokal (z. B. Gurgeln),

- systemisch (auch Chemotherapeutika),

- physikalisch (z. B. Brust- und Wadenwickel, aufsteigendes Bad, Prießnitz-Wickel).

\section{Zusatzfragen »uncharakteristisches Fieber (UF)«}

- Welche Erkrankungen können zunächst wie UF beginnen, wobei sich bei der programmierten Diagnostik mit der Checkliste Nr.1 ( Übersicht 1.1, S.38) charakteristische Symptome für bestimmte Krankheiten aufdecken lassen (z. B. Pneumonie, Erysipel, Pyelitis, Appendizitis, Thrombophlebitis)?
- Indikation zur medikamentösen Antipyrese (z. B. Gefahr von Fieberkrampf, Gefahr der Dehydratation, Beeinträchtigung des Kreislaufs, Störung des Schlafes, psychomotorische Unruhe mit verstärkter Reizbarkeit und Quengeligkeit).

- Rezeptur von fiebersenkenden Mitteln (Antipyretika) für Säuglinge, Kleinkinder, Schulkinder und Erwachsene (Wirkstoff, Darreichungsform, Dosierung, Beispiel für ein Handelspräparat).

- Diskussion der möglichen (seltenen) Problematik bei der Gabe von Acetylsalicylsäure (ASS) und Paracetamol bei Kindern und Jugendlichen im Rahmen von virusbedingten Infektionen, wie Windpocken, Masern, „Grippe« (Achtung: Reye-Syndrom: Benommenheit, Leberversagen). Acetylsalicylsäure bei Kindern dagegen ist unproblematisch z. B. gegen Rheuma oder Kopfschmerzen.

- Physikalische und allgemeine Maßnahmen (z. B. vermehrte Flüssigkeitszufuhr, leichte Bekleidung oder halbnackt, angenehme Zimmertemperatur, handwarme Waschungen oder Bäder; 5- bis 10-minütige nicht zu kalte Wickel; Bettruhe in kühlem Raum; Führung einer Fieberkurve).

- Sinn und Unsinn des ungezielten Einsatzes von Antibiotika.

- Diskussion des Einsatzes von Immunstimulanzien (z. B. Echinacea angustifolia).

- Weitere Beispiele für schweißtreibende und/oder resistenzsteigernde Heilpflanzen, wie Holunder (Flores sambuci), Lindenblüten (Flores tiliae), Wermut (Artemisia absinthum), Sonnenhut (Echinacea purpurea und angustifolia).

- Fieber als Risiko (z. B. Fieberkrampf, Exsikkose bei Kindern und alten Menschen); Fieber in der Schwangerschaft, bei alten Menschen, bei Epileptikern.

- Beratungsproblem Influenza: besonders gefährdeter Personenkreis, Diskussion der Grippeschutzimpfung, Komplikationen (Grippepneumonie und Grippeotitis). Der Neuraminidaseinhibitor (Relenza, Zanamivir) kann die Dauer der Symptome im Mittel um einen Tag verkürzen.

- Beratungsproblem Arzneimittelfieber (»drug fever«) z. B. bei H1-Rezeptoren-Blockern (»Antihistaminika») und H2-Rezeptoren-Blockern (wie Cimetidin), ferner bei »Angiotensin-converting-enzyme- (ACE-)Hemmstoffen«, wie Captopril, ferner bei Chinidin, Goldverbindungen, Heparin, Imidazolderivaten, wie den Thyreostatika Carbimazol und Thiamazol, ferner bei dem Antiarrhythmikum Procainamid. 
- Beratungsproblem Hitzschlag (Hyperthermie) bei direkter Sonnenlichteinstrahlung: rektale Temperatur kann über $41^{\circ} \mathrm{C}$ steigen (chronisch Kranke, z. B. Diabetiker, Alkoholiker, besonders gefährdet).

- Diskussion der Devise «Senkung von Fieber um jeden Preis«.

- Diskussion der Laienmeinung: „Das Fieber herauskommen lassen«. (Thermolabilität der Viren!?)

\section{Zusatzfragen »afebriler Husten«}

- Hausmittel; ärztliche Empfehlungen, wie Inhalation, Hustenbonbons (durch Aktivierung des häufigen Schluckens Hemmung des Hustens), Einreibungen, frische Luft, viel trinken.

- Rezepturen von Hustenmitteln (Antitussiva und Expektoranzien) für Säuglinge, Kinder und Erwachsene (Wirkstoff, Darreichungsform, Dosierung, Beispiel für ein Handelspräparat); Problematik von Kombinationspräparaten aus Hustenblocker und Expektorans.

- Beispiele für pflanzliche Expektoranzien (z. B. Thymian, Primula, Drosera, Mentha, Castanea, Anis, Süßholz, Wegerich, Eukalyptus).

- Charakterisierung des Opiumalkaloids Kodein: zuverlässige antitussive Wirkung, jedoch Verstopfung und (geringe) Gefahr der Abhängigkeit; relativ schwach zentral wirksames Analgetikum.

- Mögliche Problematik von ACE-Hemmern und Husten.

- Hartnäckiger Husten als Begleitsymptom einer Linksherzinsuffizienz mit dem Leitsymptom Atemnot: verursacht durch Zunahme des Blutvolumens im Lungenkreislauf um ca. 20-30\% bei horizontaler Lage.

\section{Zusatzfragen »Tonsillitis»}

- Rachenabstrich: Indikation, Durchführung, Aussagekraft, Interpretation.

- Ungefähre Häufigkeit primärer viraler Affektionen bei Kindern? (Zirka 90\%), überwiegender bakterieller Erreger? (Zirka 80\% $\beta$-hämolysierende Streptokokken).

- Suppurative Komplikationen (Peritonsillarabszess, Phlegmone, Sepsis). Nichtsuppurative Komplikationen (rheumatisches Fieber, Glomerulonephritis).

- Fraglicher Nutzen von Lokalantibiotika (z. B. Bacitracin, Tyrothricin, Fusafungin/Locabiosol).

- Zusammenhang von »Halsschmerzen« und »Bauchschmerzen« bei Kindern?!
- Antibiotische Behandlung: Notwendigkeit, Behandlungsdauer (10 Tage Penicillin oder 5 Tage Erythromycin-Estolat/Infectomycin) und Dosierung mit Standardantibiotika, Problematik der ungezielten antibiotischen Therapie.

- Urinkontrolle nach abgeschlossener Behandlung: Warum und wann?

- Indikation zur Tonsillektomie, auch in Abhängigkeit vom Lebensalter (z. B. bei wiederholten Rezidiven, chronischer Tonsillitis, Peritonsillarabszess, bei mechanisch wirksamer Hyperplasie).

- Beratung des Patienten (bzw. der Eltern) im Hinblick auf eine Operation: Funktion der Tonsillen? Stationäre Operation: Mindestens 1 Woche (mögliche Nachblutung am 1. oder 5. ostoperativen Tag!). Kontraindikationen?

- Angina Plaut-Vincent: Lokalisation und Präsentation (häufig einseitig und ulzerös).

\section{Zusatzfragen »Heiserkeit»}

- Allgemeine Empfehlungen (z. B. Flüstersprache, Vermeidung von rauchgeschwängerten Räumen).

- Wann überweisen?

- Diskussion der Refluxösophagitis als Ursache.

\section{Zusatzfragen "Schnupfen"}

- Rezepturen von lokal abschwellenden Handelspräparaten bei Säuglingen, Kindern und Erwachsenen, systemisch und lokal.

- Problematik des Abusus bestimmter Nasensprays (»Privinismus«).

- Empfehlungen zur Behandlung der chronisch-trockenen Nase (z. B. Raumklima in Büro und Schlafzimmer, Nasenpflege mit weicher Salbe, Wasser oder Emser Sole).

\section{Zusatzfragen »kombinierte Luftwegekatarrhe»}

- Diskussion des infektanfälligen Kindes (»catarrhal child «): tolerierbare Häufigkeit der fieberhaften Infekte ohne Grunderkrankung pro Jahr (ca. 6- bis 8mal); Steigerung der körperlichen Abwehrkraft (z. B. durch Klimakuren, Abhärtung, Hyperimmunglobulin), Immunstimulanzien.

- Inhalationsbehandlung mithilfe von Verdampfung und Vernebelung (unterschiedliche Teilchengröße des Aerosols). 


\section{Zusatzfragen »Kruppbilder«}

- Telefonische Erstanweisung. (Was sagt die Mutter? Was rät der Arzt?)

- Ärztliche Notfallmaßnahmen:

- allgemein (z. B. Beruhigung der Mutter, Anfeuchtung der Raumluft durch einströmendes Badewannenwasser, Absenkung der Raumtemperatur);

- speziell (Wirkstoffe/Medikamente, Dosierung, Darreichungsformen, Einweisung?)

- Rolle von Kortison (z. B. Rectodelt 100-mg-Supp.) und/oder Sauerstoff bei Laryngotracheitis und Epiglottitis.

- Mögliche Bedeutung von Schadstoffen in der Luft bei Laryngotracheitis.

- Bevorzugt betroffener Patientenkreis für Laryngotracheitis:

- "neurovegetativ labile« Kinder;

- konstitutionelle und/oder allergische Faktoren?

- Larynxfremdkörper als »Fallstrick« (immer daran denken: Patientenangaben unverlässlich!).

- Problematik der Mundinspektion mit dem Spatel bei schwerer Dyspnoe und Stridor sowie Tachykardie über 16o/min: Gefahr des reflektorischen Herz-AtemStillstands!

\section{Zusatzfragen »Impffieber«}

- Prinzipiell nach allen Impfungen möglich!

- Risikogruppen von Impflingen (Alter, Erkrankungen des Nervensystems, Schwangerschaft, geschwächte Abwehrlage, akute Erkrankung, Behandlung mit Kortikosteroiden) im Hinblick auf die öffentlich empfohlenen Impfungen.

\section{Zusatzfragen "Rezidivierende Infekte»}

- Erörterung von Beispielen aus der Praxis (z. B. infektanfälliges Kind, rezidivierender Herpes simplex, rezidivierende Bronchitis, rezidivierende Nasennebenhöhlenaffektionen).

- Erörterung der unspezifischen Klimareiztherapie (z.B. Nordsee- und Hochgebirgsklima); Gabe von $\gamma$-Globulinen; Eigenblutbehandlung als Beispiel für Erfahrungsheilkunde; Bakterienlysate wie BronchoVaxom und Uro-Vaxom. 